\title{
Erratum
}

\section{Critical droplets near coexistence of wet and nonwet surface states}

\author{
R. Bausch, R. Blossey \\ Institut für Theoretische Physik IV, Heinrich-Heine-Universität Düsseldorf, Universitätsstrasse 1, W-4000 Düsseldorf 1, Germany
}

Z. Phys. B86, 273-275 (1992)

On page 274, third line from below, the expressions for $s(f)$ and $r(f)$ have to be replaced by the single relation $f-f_{0} \sim r^{-(d-2) / 2} \exp \left(-\left(V^{\prime \prime}\left(f_{0}\right)\right)^{1 / 2} r\right)$ which has no effect on any of our conclusions.

A second correction concerns our results (3), (4), and (5), and leads to modifications of the discussions in the last two paragraphs of our paper. As they stand Eqs. (3), (4), (5) are correct only for $\sigma<3$. This is due to the singular behaviour of the slope of the scaled droplet profile $\tau(\varphi) \sim \varphi^{(1-\sigma) / 2}$ near the boundary value $\varphi_{0} \equiv f_{0} / f_{1}$ in the limit $f_{1} \rightarrow \infty$. There is a general warning by Joanny and de Gennes against a macroscopic theory e.g. of the line tension for $\sigma>3$ [1]. Nevertheless, within our model one can calculate the macroscopic behaviour of the quantities $F, R$, and $E$ for $\sigma \geqq 3$. One finds $R \sim F^{(\sigma+1) / 2}$ for all values of $\sigma$,

$$
\begin{aligned}
& F \sim S^{-1 / 2}(-\ln S)^{1 / 2} \text { for } \sigma=3, \\
& F \sim S^{-2 /(\sigma+1)} \quad \text { for } \sigma>3 \text {, } \\
& E \sim R^{d-2} \ln R \quad \text { for } \quad \sigma=3, \\
& E \sim R^{d-2} \quad \text { for } \sigma>3 \text {, }
\end{aligned}
$$

and $F \sim-\ln S, R \sim S^{-1}$ for short-range interactions $(\sigma \rightarrow \infty)$. Equations (5a) and (5b) are in agreement with recent results for the line tension by Indekeu [2]. Since from these two equations we conclude that a first-order wetting transition does not exist for $d<2, \sigma \geqq 3$ we have written the results (3a) and (3b) for $d>2$ only. For $d=2$, $\sigma=3$ Kroll, Lipowsky and Zia [3] pointed out the existence of a first-order transition in which case we expect $F \sim S^{-1 / 2}, E \sim \ln R$.

Finally, we like to emphasize the mean-field character of our results. Thermal fluctuations might indeed lead to modifications of the above picture. In our paper we already mentioned the conjecture [3] that a wetting transition is driven to second order in the so-called strong fluctuation regime $d<d_{0} \equiv(3 \sigma-1) /(\sigma+1)$. More recent renormalization group calculations [4], however, seem to allow the existence of first-order wetting transitions in the strong fluctuation regime above $d \approx 2.4$.

We are grateful to M. Lässig and R. Lipowsky for a discussion on this point.

\section{References}

1. Joanny, J.F., de Gennes, P.G.: J. Colloid Interface Sci. 111, 94 (1986)

2. Indekeu, J.O.: Physica A183, 439 (1992)

3. Kroll, D.M., Lipowsky, R., Zia, R.K.P.: Phys. Rev. B27, 1862 (1985)

4. Grotehans, S., Lipowsky, R.: Phys. Rev. A41, 4574 (1990) 